\title{
Question for Euler-Lagrange type simulator from a view point of macroscopic properties of gas-liquid multiphase flow
}

\author{
K. Ueyama \\ Department of Environmental and Energy Chemistry, \\ Faculty of Engineering, Kogakuin University, Tokyo, Japan
}

\begin{abstract}
Corrections to the conventional two-fluid model equations are briefly illustrated, showing the general expression of the two-fluid model equation without any restriction on averaging, the general expression of the interaction term with 'inside-out' property, and the corrected two-fluid model equation with a new and novel term reflecting the 'inside-out' property of the interaction term.

On the basis of the corrected two-fluid model equation, several questions are stated and discussed for Euler-Lagrange type simulator with respect to the moving velocity of individual drop and fluctuating motion of drops, and new concepts for Euler-Lagrange and Euler-Euler type of simulators are proposed focusing on the fluctuating motion of drops, which is one of the representative phenomena in the dispersed multiphase flow.
\end{abstract}

Keywords: fluctuating motion of drops, dispersion of drops, Euler-Lagrange type simulator, Euler-Euler type simulator.

\section{Introduction}

Recently, a flaw in the basis of the conventional two-fluid model equation based on volume-averaging was revealed [8]. It was shown that the restriction, that the control volume must be between the drop size and the scale of macroscopic variations, was unrealistic for treating multiphase flow with spatial variation of macroscopic properties, and the two-fluid equation has been derived without any restriction on averaging, by combining volume-averaging and time-averaging. The most important founding in the work is that the interaction term has "inside- 
out" property, which has been over looked for more than 40 years since the appearance of the two-fluid model equations based on volume-averaging $[1,9]$. The "inside-out" property causes significant corrections to the conventional twofluid model equations.

This work comprises three sections. In the first section, the derivation of the corrected two-fluid model equation is briefly illustrated. In the second section, questions for the previous Euler-Lagrange type simulators are stated and discussed with respect to the moving velocity of each drop obtained in the simulator, and the contribution of fluctuating motion of drops to the macroscopic property of the multiphase flow. In the third section, new concepts for EulerLagrange type and Euler-Euler type simulators are proposed focusing on the contribution of fluctuating motion of drops on the controlling mechanism of spatial distribution of the dispersed phase.

\section{Revision for the two-fluid model equation [8]}

\subsection{Interaction term without any restriction on averaging}

Supposing dispersed two phase flow, eqn. (1) was obtained by successively volume-averaging and time-averaging the Navier-Stokes equation for the continuous phase in an entire volume of the continuous phase in a control volume $V$.

$$
\begin{aligned}
& \frac{\partial}{\partial t}\left\{(1-\varepsilon){\overline{\rho_{c} \mathbf{u}_{c}}}^{c}\right\}+\nabla \cdot\left\{(1-\varepsilon){\overline{\rho_{c} \mathbf{u}_{c} \mathbf{u}_{c}}}^{c}\right\}-(1-\varepsilon){\overline{\rho_{c}}}^{c} \mathbf{g}+\nabla\left\{(1-\varepsilon){\overline{P_{c}}}^{c}\right\}+\nabla \cdot\left\{(1-\varepsilon) \overline{\boldsymbol{\tau}}_{c}{ }^{c}\right\}(1) \\
= & -\lim _{V \rightarrow \mathbf{X}_{0}} \frac{1}{V} \overline{\mathbf{F}}_{R, S}^{V} \Lambda
\end{aligned}
$$

Here, $\rho_{c}, \mathbf{u}_{c}, P_{c}$ and $\boldsymbol{\tau}_{c}$ are density, velocity vector, static pressure and stress tensor, respectively, of the continuous phase. A notation $\mathbf{F}_{R, S}^{V}$ on the right hand side is a sum of forces acting on drop surfaces in the control volume, and notations $\frac{-\Lambda}{q}$ and $\frac{-c}{q}$ mean time-averaged values of physical quantity $q$ for total time length of averaging $\Lambda$ and total time length during which the continuous phase covers the point of interesting $\Lambda_{c}$, respectively, as defined by eqns. (2) and (3).

$$
\begin{aligned}
& -\Lambda(\mathbf{X}, t)=\frac{1}{\Lambda} \int_{t-\frac{1}{2}}^{t+\frac{\Lambda}{2}} q(\mathbf{X}, t) d t \\
& \bar{q}^{c}(\mathbf{X}, t)=\frac{1}{\Lambda_{c}} \int_{\Lambda_{c}} q(\mathbf{X}, t) d t
\end{aligned}
$$

A notation $\mathbf{X}$ is a position vector and a notation $\int q(\mathbf{X}, t) d t$ means time $\Lambda_{c}$

integral of $q(\mathbf{X}, t)$ for duration when the point $\mathbf{X}$ is covered by the continuous 
phase. A notation $\varepsilon$ in eqn. (1) is a local hold up of the dispersed phase defined as a ratio of $\Lambda_{d}$ to the total time-averaging duration $\Lambda$, where $\Lambda_{d}$ is a total tine duration during which the point of interest is occupied by the dispersed phase. Hence, $(1-\varepsilon)$, in eqn. (1) is a local holdup of continuous phase. Following relations hold, where suffices $c$ and $d$ mean continuous and dispersed phase, respectively.

$$
\begin{gathered}
\Lambda=\Lambda_{c}+\Lambda_{d} \\
\varepsilon=\frac{\Lambda_{d}}{\Lambda} \\
\bar{q}_{c}^{\Lambda}(\mathbf{X}, t)=(1-\varepsilon){\overline{q_{c}}}^{c} \mathbf{X}(\mathbf{x}, t) \\
{\overline{q_{d}}}^{\Lambda}(\mathbf{X}, t)=\frac{1}{\Lambda} \int_{t-\frac{1}{2}}^{t+\frac{\Lambda}{2} q_{d}}(\mathbf{X}, t) d t=\frac{1}{\Lambda} \int_{\Lambda_{d}} q_{d}(\mathbf{X}, t) d t=\varepsilon{\overline{q_{d}}}^{d}(\mathbf{X}, t)
\end{gathered}
$$

A notation $\lim _{V \rightarrow \mathbf{x}_{0}}$ on the right hand side of eqn.(1) means to take a limit value when the control volume converges to a point $\mathbf{X}_{0}$. Terms on the left hand side of eqn. (1) are given at a point $\mathbf{X}_{0}$ to which the control volume $V$ converges. The right hand side of eqn. (1) is the interaction term without any restriction on averaging. Eqn. (1) shows that the interaction term in the two-fluid model equations based on time-averaging is time-averaged force acting on drop surfaces in an infinitesimal size of control volume.

\section{2 'Inside-out' property of interaction term}

The value of

$$
{\overline{\mathbf{F}_{R, S}^{V}}}^{\Lambda}=\frac{1}{\Lambda} \int_{t-\frac{1}{2}}^{t+\frac{1}{2}} \mathbf{F}_{R, S}^{V} d t
$$

for a control volume sufficiently smaller than a drop size was rigorously analysed for the dispersed two-phase flow with homogeneous size of drops with radius $R$, and eqn. (8) was obtained as a limit for the infinitesimal control volume.

$$
\begin{aligned}
& \lim _{V \rightarrow \mathbf{X}_{0}} \frac{1}{V} \overline{\mathbf{F}}_{R, S}^{V} \Lambda \\
= & -\frac{1}{\Lambda} \int_{0}^{2 \pi} d \phi \int_{0}^{\pi} d \theta\left[\sin \theta \mathbf{n}(\theta, \phi) \cdot \overline{\Delta \mathbf{T}_{c}^{\Lambda}}\left(\mathbf{X}_{0}-R \mathbf{n}(\theta, \phi): R, \theta, \phi\right) \lambda_{R}\left(\mathbf{X}_{0}-R \mathbf{n}(\theta, \phi)\right) R^{2}\right] \\
& -\frac{1}{\Lambda} \int_{0}^{2 \pi} d \phi \int_{0}^{\pi} d \theta\left[\sin \theta \mathbf{n}(\theta, \phi) \cdot \overline{\mathbf{T}}_{c}^{c}\left(\mathbf{x}_{0}\right) \lambda_{R}\left(\mathbf{X}_{0}-R \mathbf{n}(\theta, \phi)\right) R^{2}\right]
\end{aligned}
$$


Here, $\mathbf{T}_{c}(\mathbf{X}: R, \theta, \phi)$ is defined by eqn. (9) below, where $\mathbf{I}$ denotes the unit tensor: A notation $(\mathbf{X}: R, \theta, \phi)$ means a position in a spherical coordinate system $\{r, \theta, \phi\}$ with its origin at point $\mathbf{X}$. Notations $\mathbf{n}(\theta, \phi)$ and $\lambda_{R}(\mathbf{X})$ are

$$
\mathbf{T}_{c}(\mathbf{X}: R, \theta, \phi)=P_{c}(\mathbf{X}: R, \theta, \phi) \mathbf{I}+\boldsymbol{\tau}_{c}(\mathbf{X}: R, \theta, \phi)
$$

unit vector on the $r$-axis to the direction $(\theta, \phi)$ and a duration in which the center of a drop with radius $R$ is in unit volume assigned at point $\mathbf{X}$, respectively. A notation $\Delta \mathbf{T}_{c}^{\Lambda}$ is the difference between time-averaged values of $\mathbf{T}_{c}$ at angular coordinates $(\theta, \phi)$ on the surface, which is $\overline{\mathbf{T}_{c}^{\Lambda}}(\mathbf{X}-R \mathbf{n}(\theta, \phi): R, \theta, \phi)$, and that at the same position in the bulk, which is $\overline{\mathbf{T}}_{c}^{c}(\mathbf{X})$, as defined by eqn. (10), which was introduced to utilize the knowledge on the drag force, lift force and virtual mass

$$
\Delta \overline{\mathbf{T}_{c}^{\Lambda}}(\mathbf{X}-R \mathbf{n}(\theta, \phi): R, \theta, \phi)=\overline{\mathbf{T}_{c}^{\Lambda}}(\mathbf{X}-R \mathbf{n}(\theta, \phi): R, \theta, \phi)-{\overline{\mathbf{T}_{c}}}^{c}(\mathbf{X})
$$

force. The sum of them, $\overline{\mathbf{f}_{d}^{\Delta \mathbf{T}}}\left(\mathbf{X}_{0}\right)$, is given by eqn. (11). Knowledge on the drag force, lift force and virtual mass force can be applied to $\overline{\mathbf{f}_{d}^{\Delta \mathbf{T}}}$.

$$
\overline{\mathbf{f}_{d}^{\Delta \mathbf{T}}}\left(\mathbf{X}_{0}\right)=-\int_{0}^{2 \pi} d \phi \int_{0}^{\pi} d \theta\left[\sin \theta \mathbf{n}(\theta, \phi) \cdot \Delta \overline{\mathbf{T}_{c}^{\Lambda}}\left(\mathbf{X}_{0}: R, \theta, \phi\right) R^{2}\right]
$$

To consider the first term on the right hand side of eqn. (8), the positions where the values of $\Delta \mathbf{T}_{c}^{\Lambda}$ and $\lambda_{R}$ are specified were exchanged. That is;

$$
\begin{aligned}
& -\frac{1}{\Lambda} \int_{0}^{2 \pi} d \phi \int_{0}^{\pi} d \theta\left[\sin \theta \mathbf{n}(\theta, \phi) \cdot \Delta \overline{\mathbf{T}_{c}^{\Lambda}}\left(\mathbf{X}_{0}: R, \theta, \phi\right) \lambda_{R}\left(\mathbf{X}_{0}\right) R^{2}\right] \\
= & -\frac{\lambda_{R}\left(\mathbf{X}_{0}\right)}{\Lambda} \int_{0}^{2 \pi} d \phi \int_{0}^{\pi} d \theta\left[\sin \theta \mathbf{n}(\theta, \phi) \cdot \Delta \overline{\mathbf{T}_{c}^{\Lambda}}\left(\mathbf{X}_{0}: R, \theta, \phi\right) R^{2}\right] \\
= & \frac{V_{d} \lambda_{R}\left(\mathbf{X}_{0}\right)}{\Lambda} \frac{1}{V_{d}} \overline{\mathbf{f}_{d}^{\Delta \mathrm{T}}}\left(\mathbf{X}_{0}\right) \approx \frac{\Lambda_{d}\left(\mathbf{X}_{0}\right)}{\Lambda} \frac{1}{V_{d}} \overline{\mathbf{f}_{d}^{\Delta \mathrm{T}}}\left(\mathbf{X}_{0}\right)=\varepsilon\left(\mathbf{X}_{0}\right) \frac{1}{V_{d}} \overline{\mathbf{f}_{d}^{\Delta \mathrm{T}}}\left(\mathbf{X}_{0}\right)
\end{aligned}
$$

The last expression on the right hand side of eqn. (12) is the same as the conventional interaction term. The intrinsic properties of the interaction term in the two-fluid model equations is visible in eqn. (8), which differs clearly from the conventional interaction term in its 'inside-out' property.

\subsection{Revised two-fluid model equation}

The 'inside-out' property can be treated by using Taylor expansion to shift the points where $\Delta \overline{\mathbf{T}}_{c}{ }^{c}$ and $\lambda_{R}$ are specified. Upon neglecting higher order terms, eqn (13) was finally obtained.

$$
\begin{aligned}
& \lim _{V \rightarrow \mathbf{x}_{0}} \frac{1}{V} \overline{\mathbf{F}_{R, S}^{V}} \Lambda \\
\approx & \varepsilon\left(\mathbf{X}_{0}\right) \frac{\overline{\mathbf{f}_{d}^{\Delta \mathbf{T}}}\left(\mathbf{X}_{0}\right)}{V_{d}}-\left.c R\left(\frac{\partial \varepsilon}{\partial Z}\right)\right|_{\mathbf{X}_{0}} \frac{\overline{\mathbf{f}_{d}^{\Delta \mathbf{T}+}}\left(\mathbf{X}_{0}\right)-\overline{\mathbf{f}_{d}^{\Delta \mathbf{T}}-}\left(\mathbf{X}_{0}\right)}{V_{d}}+\left.(\nabla \varepsilon)\right|_{\mathbf{X}_{0}} \overline{P_{c}^{c}}\left(\mathbf{X}_{0}\right)+\left.(\nabla \varepsilon)\right|_{\mathbf{X}_{0}} \cdot \overline{\boldsymbol{\tau}_{c}^{c}}\left(\mathbf{X}_{0}\right)
\end{aligned}
$$


The first term on the right hand side of eqn. (13) is the same as the conventional interaction term. Standard analyses of the drag force, lift force and virtual mass force for a single drop in a flow field can now be applied to $\overline{\mathbf{f}_{d}^{\Delta \mathbf{T}}}\left(\mathbf{X}_{0}\right)$.

The second, third and fourth terms in eqn. (13) was introduced to compensate for the discrepancy between $\lim _{V \rightarrow \mathbf{x}_{0}} \frac{1}{V} \overline{\mathbf{F}}_{R, S}^{V} \Lambda$

and the conventional interaction term $\varepsilon\left(\mathbf{X}_{0}\right) \frac{\overline{\mathbf{f}_{d}^{\Delta \mathrm{T}}}\left(\mathbf{X}_{0}\right)}{V_{d}}$.

The $Z$ axis in the second term on the right hand side of eqn. (13) is chosen to the direction of $\nabla \varepsilon$, and $\overline{\mathbf{f}_{d}^{\Delta \mathbf{T}}+}\left(\mathbf{X}_{0}\right)$ and $\overline{\mathbf{f}_{d}^{\Delta \mathbf{T}-}}\left(\mathbf{X}_{0}\right)$ are forces acting on the two hemispherical surfaces of the drop having positive and negative values of $Z$ respectively. The parameter $c$ represents the effects of the weight function, $\cos \theta$, in the surface integration over the hemisphere surface, and is between 0 and 1 . This term becomes significant for flow having steep gradients of the local holdup and averaged velocity.

The third term on the right-hand side of eqn. (13) is similar to the result of Kolev [4] in rearranging surface integrals for two-fluid model equations based on volume-averaging, which is crucial to excluding local holdup from the differential operator $\nabla$ in the static pressure term in the two-fluid model equation.

The fourth term on the right hand side of eqn. (13) arises from the definition of the force acting on a drop, $\overline{\mathbf{f}_{d}^{\Delta \mathbf{T}}}$, given by eqn. (11), since $\Delta \overline{\mathbf{T}_{c}^{\Lambda}}$, which is the difference between time- averaged values of tensor, $\mathbf{T}_{c}=P_{c} \mathbf{I}+\boldsymbol{\tau}_{c}$, on a drop surface and in the bulk of the continuous phase, was taken to define the force acting on a drop $\overline{\mathbf{f}_{d}^{\Delta \mathrm{T}}}$. This term makes a small modification the shear stress term in the two-fluid model equation.

Finally, the two-fluid model equation is given as eqn. (14).

$$
\begin{aligned}
& \frac{\partial}{\partial t}\left\{(1-\varepsilon) \overline{\rho_{c} \mathbf{u}_{c}}{ }^{c}\right\}+\nabla \cdot\left\{(1-\varepsilon) \overline{\rho_{c} \mathbf{u}_{c} \mathbf{u}_{c}}{ }^{c}\right\} \\
= & -\varepsilon \frac{\overline{\mathbf{f}_{d}^{\Delta \mathbf{T}}}}{V_{d}}-(1-\varepsilon)\left(\nabla \overline{P_{c}}\right)-(1-\varepsilon)\left(\nabla \cdot \overline{\boldsymbol{\tau}}_{c}^{c}\right)+(1-\varepsilon){\overline{\rho_{c}}}^{c} \mathbf{g}+c R\left(\frac{\partial \varepsilon}{\partial Z}\right) \frac{\overline{\mathbf{f}_{d}^{\Delta \mathbf{T}+}}\left(\mathbf{X}_{0}\right)-\overline{\mathbf{f}_{d}^{\Delta \mathbf{T}-}}\left(\mathbf{x}_{0}\right)}{V_{d}}
\end{aligned}
$$

Eqn. (14) is derived without any restriction on averaging, to which the accumulated knowledge on the drag force, lift force and virtual mass force can be applied.

\section{Question for Euler-Lagrange type simulator}

In the preceding section, it has been revealed that the conventional two-fluid model equations based on volume-averaging passed over the "inside-out" property of the interaction term due to the restriction on the size of the control volume. It has been also shown that we have eqn. (14) as the two-fluid model equation without any restriction on averaging, to which the accumulated knowledge on the drag force, lift force and virtual mass force can be applied. It 
is worth noting here that eqn. (14) is obtained by combining volume-averaging and time-averaging, and the result, eqn. (14), is based on time-averaging. This means that we have to keep in mind that the averaged physical quantities in the two-fluid model equation are to be based on time-averaging. In this section, several questions for the conventional Euler-Lagrange type simulators are made and discussed from a view point of macroscopic properties of gas-liquid multiphase flow.

\subsection{Over-view of dispersed two-phase flow and questions for Euler- Lagrange type simulator}

In a bubble column operated in the recirculating turbulent flow regime, it is well known that the flow field in the columns is fully developed turbulent flow with distributed size of vortices up to the order of column diameter [3,7]. Bubbles are rising in highly turbulent liquid flow with considerable scale of fluctuating motion induced by the vortices. The well-known parabolic distribution of gas holdup in bubble columns operated in this flow regime $[3,7]$ has been a hot target for numerical studies, and has not been successively realized yet [10]. The reason why bubbles are gathering in the central region of the column may be attributed to the lift force, and almost all of the previous works have been focused on the force acting on a drop, such as Tomiyama coefficient for the lift force [5], the dispersion force [2], and so on.

In Euler-Lagrange type simulators, a trail of each drop is followed by an equation of motion for each drop, to which accumulated knowledge on the forces acting on a drop is applied. The values of moving velocity of drops thus obtained are "averaged" values, because the flow field of the surrounding fluid described by the two-fluid model equation is averaged one. Several questions arise here: What kind of "mean" value is the moving velocity obtained from the equation of motion? Can we ignore the contribution of fluctuating motion of drops to the macroscopic property of the multiphase flow? What kind of forces should be accounted on the equation of motion for a drop? Let us discuss these questions next.

\subsection{Equation of motion for a drop}

The equation of motion for a drop is given by Newton's law, which is a balance between the acceleration term and the sum of forces acting on the drop. The force acting on an entire surface of a drop is the contribution of a tensor $\mathbf{T}_{c}$ acting on the drop surface, however, the sum of the drag force, lift force and virtual mass force is given by eqn (11) as $\overline{\mathbf{f}_{d}^{4 T}}\left(\mathbf{x}_{0}\right)$ which is assigned for the contribution of $\Delta \overline{\mathbf{T}_{c}^{\Lambda}}$ on the drop surface. Hence, the contribution of $\overline{\mathbf{T}_{c}^{\Lambda}}\left(\mathbf{X}_{0}: R, \theta, \phi\right)-\Delta \overline{\mathbf{T}_{c}^{\Lambda}}\left(\mathbf{X}_{0}: R, \theta, \phi\right)=\overline{\mathbf{T}}_{c}^{c}\left(\mathbf{X}_{0}+R \mathbf{n}(\theta, \phi)\right)$ should be accounted in addition to the force $\overline{\mathbf{f}_{d}^{\mathrm{T}}}\left(\mathbf{x}_{0}\right)$, to account all the forces acting on the drop surface. That is; 


$$
\begin{aligned}
\overline{\mathbf{f}_{b}}\left(\mathbf{X}_{0}\right) & =-\int_{0}^{2 \pi} d \phi \int_{0}^{\pi} d \theta\left[\sin \theta \mathbf{n}(\theta, \phi) \cdot \overline{\mathbf{T}}_{c}^{c}\left(\mathbf{X}_{0}+R \mathbf{n}\right) R^{2}\right] \\
& =-\int_{V d} \nabla \cdot\left(\bar{P}_{c}^{c} \mathbf{I}+\overline{\boldsymbol{\tau}}_{c}^{c}\right) d V \approx V_{d}\left(\nabla \overline{P_{c}}\right)_{\mathbf{X}_{0}}+V_{d}\left(\nabla \cdot \overline{\boldsymbol{\tau}_{c}}\right)_{\mathbf{X}_{0}}
\end{aligned}
$$

Here, $V_{d}$ is volume of a drop and $\int_{V_{d}} d V$ means an integration over $V_{d}$. The force $\overline{\mathbf{f}_{b}}\left(\mathbf{X}_{0}\right)$ given by eqn. (15) is regarded as buoyancy force, because the second term on the right hand side of the last expression in eqn. (15) can be neglected comparing with the first term.

The accumulated knowledge on the drag force, lift force and virtual mass force tells that the sum of those forces can be expressed as a function of liquid velocity $\mathbf{u}_{c}$ and moving velocity of a drop $\mathbf{u}_{p}$, as eqn. (16). The function $H$ can

$$
\mathbf{f}_{d}^{\Delta \mathbf{T}}=H\left(\mathbf{u}_{c}, \mathbf{u}_{p}\right)
$$

be easily found in text books or literatures in this research field. Since we have an intention to utilize the two-fluid model equation, which is described in terms of time-averaged physical quantities, together with eqn. (16), let us expect that eqn (17) hold for the time-averaged velocity field of liquid ${\overline{\mathbf{u}_{c}}}_{c}$ and averaged moving velocity of a drop $\overline{\mathbf{u}_{p}}$.

$$
\overline{\mathbf{f}_{d}^{\Delta \mathbf{T}}}=H\left(\overline{\mathbf{u}_{c}}, \overline{\mathbf{u}_{p}}\right)
$$

Here, $\overline{\mathbf{f}_{d}^{\mathbf{T}}}\left(\mathbf{x}_{0}\right)$ is a sum of the drag force, lift force and virtual mass force acting on a drop defined by eqn. (11). The averaged moving velocity of a drop $\overline{\mathbf{u}_{p}}$ is regarded as moving velocity of a drop defined by eqn. (17) for the flow field of the time-averaged velocity of the continuous phase $\overline{\mathbf{u}}_{c}^{c}$.

Now, we have eqn. (18) as the equation of motion for a drop.

$$
\frac{d}{d t}\left(\rho_{d} V_{d} \overline{\mathbf{u}_{p}}\right)=H\left(\overline{\mathbf{u}_{c}}, \overline{\mathbf{u}_{p}}\right)+\overline{\mathbf{f}_{\mathrm{b}}}+\rho_{d} V_{d} \mathbf{g}
$$

Here, $\overline{\mathbf{f}}_{b}$ is buoyancy force acting on the drop.

It is worth noting here that the dispersion force [2] does not appear on the right hand side of eqn. (18) because it was introduced in modifying Reynolds stress in the second term on the left hand side of eqn. (14). The term is acceptable in the two-fluid model equation, but does not appear in the equation of motion for a drop.

\section{Proposal for Euler-Lagrange and Euler-Euler types of simulators}

The second term on the left hand side of eqn. (14) can be expressed in terms of time-averaged velocity field of continuous phase by using $\kappa-\varepsilon$ model, for instance, probably with the dispersion force. The fifth term on the right hand 
side of eqn. (14) cannot be treated at present due to the lack of knowledge, however, it should be resolved for the practical use sooner or later. Let us skip to the utilization of eqn. (14) for the simulator of multiphase flow.

\subsection{Euler-Lagrange type of simulator}

Since the averaged moving velocity of a drop, $\overline{\mathbf{u}_{p}}$, is regarded as moving velocity of a drop defined by eqn. (17) for the flow field of the time-averaged velocity of the continuous phase, the actual moving velocity of a drop, $\mathbf{u}_{p}$, is given as eqn. (19).

$$
\mathbf{u}_{p}=\overline{\mathbf{u}_{p}}+\mathbf{u}_{p}^{\prime}
$$

Here, $\mathbf{u}_{p}^{\prime}$ is fluctuating component of moving velocity of a drop induced by turbulent vortices in surrounding fluid, which should play a key role in the simulator to control the distribution of local holdup of the dispersed phase through dispersion phenomena of drops.

Eqns. (14) and (18), and eqn. (20) which follows are basic equations for the Euler-Lagrange type of simulator.

$$
\rho_{c} \frac{\partial}{\partial t}(1-\varepsilon)+\nabla \cdot\left\{(1-\varepsilon) \overline{\mathbf{u}_{c}} c\right\}=0
$$

Eqn. (20) is the time-averaged equation of continuity for the continuous phase [6].

Supposing that spatial distributions of $\overline{\mathbf{u}}_{c}^{c}$ and ${\overline{P_{c}}}_{c}$, and averaged moving velocity, $\overline{\mathbf{u}_{p}}$, and position of individual drop are known at time $t=t_{1}$, the spatial distributions of $(1-\varepsilon){\overline{\mathbf{u}_{c}}}^{c}$ at the next time step $t=t_{1}+\Delta t$ can be calculated from eqn. (14). The position of individual drop at the next time step is given by adding $\Delta t \mathbf{u}_{p}=\Delta t\left(\overline{\mathbf{u}_{p}}+\mathbf{u}^{\prime}{ }_{p}\right)$ to the position vector of each drop, then the spatial distribution of $\varepsilon$ at $t=t_{1}+\Delta t$ can be obtained. Then, the value of $\overline{\mathbf{u}}_{c}^{c}$ at $t=t_{1}+\Delta t$ can be calculated, and the value of $\overline{\mathbf{u}_{p}}$ at the next time step $t=t_{1}+\Delta t$ is given by eqn. (18). The spatial distribution of ${\overline{P_{c}}}_{c}$ at the next time step are determined so that the spatial distributions of $\overline{\mathbf{u}}_{c}{ }^{c}$ and ${\overline{P_{c}}}^{c}$ satisfy both eqns (14) and (20).

How to give the value of the fluctuating component $\mathbf{u}_{p}^{\prime}$ to each drop is not known at present, however the trial and error approach in the numerical experiments to realize the distribution of the dispersed phase seem to be useful to obtain effective suggestion for the fluctuating motion. 


\subsection{Euler-Euler type of simulator}

The contribution of fluctuating motion of drops to the controlling mechanism of the distribution of local holdup of the dispersed phase can also be treated by introducing dispersion coefficient in stead of fluctuating velocity. Since the time-averaged velocity of the dispersed phase ${\overline{\mathbf{u}_{c}}}_{c}^{c}$ includes contributions of both averaged moving velocity of drops $\overline{\mathbf{u}_{p}}$ and dispersion of drops due to fluctuating moving velocity $\mathbf{u}_{p}^{\prime}$, we have eqn. (21).

$$
\varepsilon \overline{\mathbf{u}_{d}}=\varepsilon \overline{\mathbf{u}_{p}}-D_{\varepsilon}(\nabla \varepsilon)
$$

Here, $D_{\varepsilon}$ is a dispersion coefficient of drops. A notation $\bar{q}^{d}$ means timeaveraged values of physical quantity $q$ for total time length during which the dispersed phase covers the point of interesting.

Equation of continuity for the drop phase time-averaged for the dispersed two-phase flow is obtained as eqn. (22) [6].

$$
\frac{\partial}{\partial t} \varepsilon+\nabla \cdot\left(\varepsilon \overline{\mathbf{u}_{d}} d\right)=0
$$

Introducing eqn. (21) into eqn. (22), eqn. (23) is obtained.

$$
\frac{\partial}{\partial t} \varepsilon+\nabla \cdot\left(\varepsilon \overline{\mathbf{u}_{p}}\right)=\nabla \cdot\left\{D_{\varepsilon}(\nabla \varepsilon)\right\}
$$

Eqn. (24) also holds as the Navier-Stokes equation for the dispersed phase time-averaged for dispersed multiphase flow [6].

$$
\begin{aligned}
& \frac{\partial}{\partial t}\left(\varepsilon{\overline{\rho_{d} \mathbf{u}_{d}}}^{d}\right)+\nabla \cdot\left(\varepsilon \overline{\rho_{d} \mathbf{u}_{d} \mathbf{u}_{d}}{ }^{d}\right)-\varepsilon{\overline{\rho_{d}}}^{d} \mathbf{g}+\nabla\left\{\varepsilon \overline{P_{d}} d\right\}+\nabla \cdot\left\{\varepsilon \overline{\boldsymbol{\tau}_{d}} d\right\} \\
= & \lim _{V \rightarrow \mathbf{x}_{0}} \frac{1}{V} \overline{\mathbf{F}}_{R, S}^{V}
\end{aligned}
$$

From eqns. (14) and (24), eqn. (25) is obtained.

$$
\begin{aligned}
0= & \frac{\partial}{\partial t}\left\{(1-\varepsilon){\overline{\rho_{c} \mathbf{u}_{c}}}^{c}\right\}+\frac{\partial}{\partial t}\left(\varepsilon \overline{\rho_{d} \mathbf{u}_{d}}{ }^{d}\right)+\nabla \cdot\left\{(1-\varepsilon){\overline{\rho_{c} \mathbf{u}_{c} \mathbf{u}_{c}}}^{c}\right\}+\nabla \cdot\left(\varepsilon \overline{\rho_{d} \mathbf{u}_{d} \mathbf{u}_{d}}{ }^{d}\right) \\
& -(1-\varepsilon){\overline{\rho_{c}}}^{c} \mathbf{g}-\varepsilon \overline{\rho_{d}}{ }^{d} \mathbf{g}+\nabla{\overline{P_{c}}}^{c}+\nabla \cdot\left\{(1-\varepsilon){\overline{\boldsymbol{\tau}_{c}}}^{c}\right\}+\nabla \cdot\left\{\varepsilon \overline{\boldsymbol{\tau}_{d}}{ }^{d}\right\}
\end{aligned}
$$

Here, the values of static pressure time-averaged for dispersed and continuous phases are assumed to be the same.

Eqns. (14), (17), (20), (23) and (25) are basic equations for the Euler-Euler type simulator. Once we know the spatial distributions of time-averaged velocity of the continuous phase ${\overline{\mathbf{u}_{c}}}_{c}^{c}$, the average moving velocity of a drop $\overline{\mathbf{u}_{p}}$, time-averaged static pressure for the continuous phase ${\overline{P_{c}}}^{c}$ and local holdup of the dispersed phase $\varepsilon$, we can calculate the spatial distributions of them at the next time step, by using eqns. (14), (17), (20), (23) and (25). 
Accumulated knowledge on the dispersion coefficient $D_{\varepsilon}$ is poor at present; however, the trial and error approach in the numerical experiments to realize the distribution of the dispersed phase is an effective approach to investigate the fluctuating motion.

\section{Conclusion}

Corrections to the conventional two-fluid model equations have been briefly illustrated, showing eqn. (1) as the general expression of the two-fluid model equation without any restriction on averaging, eqn. (8) as the general expression of the interaction term with 'inside-out' property, and eqn. (14) as the corrected two-fluid model equation with a new and novel term comprising the product of the local gradient of holdup and the difference between the forces acting on a hemispherical surface of the drop cut by a plane perpendicular to the local holdup gradient[8].

Questions for the previous Euler-Lagrange type simulators have been stated and discussed with respect to the moving velocity of each drop obtained in the simulator and the contribution of fluctuating motion of drops to the macroscopic property of the multiphase flow. It has also been shown in the discussion that the contribution of fluctuating motion of drops on the macroscopic properties of multiphase flow cannot be treated in the previous Euler-Lagrange type simulator because both the two-fluid model equation and the equation of motion for a drop are relations between time-averaged velocity field of surrounding fluid and averaged moving velocity of a drop.

Concepts of Euler-Lagrange type and Euler-Euler type simulators are proposed focusing on the contribution of fluctuating motion of drops to the controlling mechanism of spatial distribution of the dispersed phase. It was shown that the fluctuating motion of drops, which cannot be treated in the twofluid model equation and the equation of motion of a drop, may play a key role in controlling the spatial distribution of local holdup of dispersed phase.

\section{Symbols}

$\begin{aligned} \frac{D_{\varepsilon}:}{\mathbf{f}_{b}:} & \text { dispersion coefficient of drops } \\ & \text { force acting on drop surface due to tensor } \mathbf{T}_{c} \text { time-averaged } \\ & \text { for bulk of the continuous phase, which nearly equals to the } \\ & \text { buoyancy force as defined by eqn. (15). } \\ & \text { contribution of the difference between the values of } \mathbf{T}_{c} \\ & \text { at the drop surface and in bulk of the surrounding fluid, } \\ & \text { on an entire surface of single drop, which is the sum of the } \\ & \text { drag, lift and virtual mass forces for a single drop in } \\ & \text { a flow field }\end{aligned}$


$\overline{\mathbf{f}_{d}^{\Delta \mathbf{T}}}: \quad$ contribution of $\Delta \overline{\mathbf{T}_{c}^{\Lambda}}$ on an entire surface of a drop, given by eqn (19) $\left\lfloor k g \cdot m \cdot s^{-2}\right\rfloor$

$\overline{\mathbf{f}_{d}^{\Delta \mathbf{T}+}}$ : force due to $\Delta \overline{\mathbf{T}_{c}^{\Lambda}}$ acting on a hemispherical surface of a drop with positive value of $Z$

$\overline{\mathbf{f}_{d}^{\Delta \mathbf{T}^{-}}}$: force due to $\Delta \overline{\mathbf{T}_{c}^{\Lambda}}$ acting on a hemispherical surface of $\left\lfloor k g \cdot m \cdot s^{-2}\right\rfloor$ a drop with negative value of $Z$ $\left\lfloor k g \cdot m \cdot s^{-2}\right\rfloor$

$\mathbf{F}_{R, S}^{V}: \quad$ sum of forces acting on drop surfaces in a control volume $\left[\mathrm{kg} \cdot \mathrm{m} \cdot \mathrm{s}^{-2}\right\rfloor$

g: vector of gravitational acceleration

I : unit tensor

$\mathbf{n}(\theta, \phi)$ : unit vector along the $r$ axis in spherical coordinate system directed to $(\theta, \phi)$

$P: \quad$ static pressure

$q: \quad$ physical quantity

$r: \quad$ radial coordinate in spherical coordinate system

$R: \quad$ radius of a drop

$S: \quad$ surface of a control volume

$t: \quad$ time

$\mathbf{T}_{c}: \quad$ tensor composed of static pressure and shear stress

tensor, defined as eqn. (9) $\left[\mathrm{kg} \cdot \mathrm{m}^{-1} \cdot \mathrm{s}^{-2}\right]$

$\overline{\mathbf{T}_{c}^{\Lambda}}$ : mean value of tensor $\mathbf{T}_{c}$ for duration $\Lambda$, at a fixed angular coordinate on drop surface

$\mathbf{u}$ : velocity vector

$\mathbf{u}_{p}$ : moving velocity vector of a drop $\left[\mathrm{kg} \cdot \mathrm{m}^{-1} \cdot \mathrm{s}^{-2}\right]$ $\left[m \cdot s^{-1}\right]$ $\left[m \cdot s^{-1}\right]$

$\overline{\mathbf{u}}_{p} \quad$ averaged moving velocity vector of a drop, defined by eqn (26) $\left[\mathrm{m} \cdot \mathrm{s}^{-1}\right]$

$\mathbf{u}_{p}^{\prime} \quad$ fluctuating moving velocity vector of a drop $\left[m \cdot s^{-1}\right]$

$V: \quad$ volume of the control volume

$V_{d}: \quad$ volume of a drop

$\mathbf{X}, \mathbf{X}_{0}$ : position vector in Cartesian coordinate

$Z: \quad$ coordinates in Cartesian coordinate system

$\Delta t: \quad$ time step in numerical calculation

$\varepsilon: \quad$ local hold up of the dispersed phase, defined by eqn (8)

$\phi, \theta: \quad$ coordinates in spherical coordinate system

$\lambda_{R}(\mathbf{X})$ : duration in which the center of a drop with radius $R$ is in unit volume assigned at point $\mathbf{X}$ 
1: $\quad$ total duration for time averaging

$\Lambda_{d}$ : total time during which the dispersed phase occupies the point of interest

$\Lambda_{c}$ : total time during which the continuous phase occupies the point of interest

$\rho: \quad$ density

$\tau$ : $\quad$ shear stress tensor

\section{Suffixes and other notations}

$c: \quad$ continuous phase

$d$ : dispersed phase

${ }^{-\Lambda}$ : time-averaged value for total duration for time-averaging, $\Lambda$

${ }^{-c}: \quad$ time-averaged value for duration $\Lambda_{c}$

${ }^{-d}$ : time-averaged value for duration $\Lambda_{d}$

\section{References}

[1] Anderson, T. B. and R. Jackson, A fluid mechanical description of fluidized bed, Ind. Eng. Fundam., 6, 527-534, 1967

[2] Bertodano, M. L., R. T. Lahey and O. C. Jones, Phase distribution in bubbly two-phase flow in vertical ducts, Int. J. Multiphase Flow, 20, 805818,1994

[3] Hills, J.H., Radial non-uniformity of velocity and voidage in a bubble column, Trans. Inst. Chem. Engrs., 52, 1-10, 1974.

[4] Kolev, N. I., Multiphase Flow Dynamics, 3rd ed., 51-54, SpringerVerlag Berlin Heidelberg, 2007

[5] Tomiyama, A., Y. Nakahara, Y. Adachi and S. Hosokawa, Shapes and rising velocity of single bubbles rising through an inner subchannel, $J$. Nuclear Science and Technology, 40, 136-142, 2003

[6] Ueyama, K. and T. Miyauchi, Time-averaged Navier-Stokes equations as Basic Equations for multiphase flow, Kagakukogaku Ronbunshu, 2, 595-601, 1976.

[7] Ueyama, K. and T. Miyauchi, Properties of recirculating turbulent two phase flow in gas bubble columns, AIChEJ., 25, 258-266, 1979.

[8] Ueyama, K., A study of two-fluid model equations, submitted to J. Fluid Mechanics

[9] Whitaker, S., Diffusion and dispersion in porous media, AIChE Journal, $13,420-427,1967$

[10] Zhang, X. and G. Ahmadi, Eulerian-Lagrangian simulations of liquidgas-solid flows in three-phase slurry reactors, Chem. Eng. Sci., 60, 50895104, 2005. 